Article

\title{
Interpolative Reich-Rus-Ćirić Type Contractions on Partial Metric Spaces
}

\author{
Erdal Karapinar $1,2, * \mathbb{C}$, Ravi Agarwal ${ }^{3}$ and Hassen Aydi $4{ }^{4}$ \\ 1 Department of Mathematics, Atilim University, Incek 06836, Ankara, Turkey \\ 2 Department of Medical Research, China Medical University Hospital, China Medical University, \\ Taichung 40402, Taiwan \\ 3 Department of Mathematics, Texas A\&M University-Kingsville, Kingsville, TX 78363, USA; \\ agarwal@tamuk.edu \\ 4 College of Education in Jubail, Department of Mathematics, Imam Abdulrahman Bin Faisal University, \\ P.O. 12020, Industrial Jubail 31961, Saudi Arabia; hmaydi@iau.edu.sa \\ * Correspondence: erdalkarapinar@yahoo.com or karapinar@mail.cmuh.org.tw
}

Received: 23 October 2018; Accepted: 14 November 2018; Published: 16 November 2018

\begin{abstract}
By giving a counter-example, we point out a gap in the paper by Karapinar (Adv. Theory Nonlinear Anal. Its Appl. 2018, 2, 85-87) where the given fixed point may be not unique and we present the corrected version. We also state the celebrated fixed point theorem of Reich-Rus-Ćirić in the framework of complete partial metric spaces, by taking the interpolation theory into account. Some examples are provided where the main result in papers by Reich (Can. Math. Bull. 1971, 14, 121-124; Boll. Unione Mat. Ital. 1972, 4, 26-42 and Boll. Unione Mat. Ital. 1971, 4, 1-11.) is not applicable.
\end{abstract}

Keywords: partial metric; interpolative Reich-Rus-Ćirić type contraction; fixed point

MSC: 46T99; 47H10; 54H25

\section{Introduction and Preliminaries}

In 1922, Banach laid the foundations of metric fixed point theory by proposing his prominent fixed point result. To put a finer point on it, Banach observed that if a self-mapping $T$, defined on complete metric space $(X, \rho)$, fulfills the contraction inequality, i.e., there exists a constant $k \in[0,1)$ such that

$$
\rho(T \xi, T \eta) \leq k \rho(\xi, \eta) \text { for all } \xi, \eta \in X,
$$

then it possesses a unique fixed point in X. In 1968, Kannan [1] proposed a new fixed point result. He considered the following contraction type:

$$
\rho(T \xi, T \eta) \leq \lambda[\rho(\xi, T \xi)+\rho(\eta, T \eta)] \text { for all } \xi, \eta \in X,
$$

where $\lambda \in\left[0, \frac{1}{2}\right)$. Very recently, in [2], the acclaimed theorem of Kannan was revisited by taking the interpolation theory into account. For a metric space $(X, \rho)$, the self-mapping $T: X \rightarrow X$ is said to be an interpolative Kannan type contraction, if there are constants $\lambda \in[0,1)$ and $\alpha \in(0,1)$ such that

$$
\rho(T \xi, T \eta) \leq \lambda[\rho(\xi, T \xi)]^{\alpha} \cdot[\rho(\eta, T \eta)]^{1-\alpha},
$$

for all $\xi, \eta \in X$ with $\xi \neq T \xi$. The main result in [2] via an interpolative Kannan type contraction is

Theorem 1 ([2]). In the framework of a complete metric space $(X, \rho)$, if a mapping $T: X \rightarrow X$ forms an interpolative Kannan type contraction, then it possesses a unique fixed point in $X$. 
Note that when the inequality (2) holds for all $\xi, \eta \in X$, and if $T$ possesses a fixed point (say $v \in X$ ), then $T \xi=v$ for each $\xi \in X$, that is, $T$ is a constant mapping, which is the trivial case, so the fixed point of $T$ is unique. The appropriate condition on $\xi$ and $\eta$ in (2) should be $\xi, \eta \in X \backslash$ Fix $(T)$, where $\operatorname{Fix}(T)=\{u \in X, T u=u\}$. In this case, the author [2] ensured the existence of a unique fixed point. There is a gap, that is, such fixed point is not necessarily unique. The following example illustrates our concern.

Example 1. Set $X=\{0,1,2,3\}$ that is endowed with the Euclidean metric $\rho(\xi, \eta)=|\xi-\eta|$. Consider

$$
T 0=0, \quad T 1=1, \quad T 2=T 3=1 .
$$

Let $\xi, \eta \in X \backslash$ Fix $(T)$. Then, $(\xi, \eta) \in\{(2,3),(3,2),(2,2),(3,3)\}$. Thus, (2) is satisfied for all $\lambda \in[0,1)$ and $\alpha \in(0,1)$. It is evident that both 0 and 1 are fixed points for the self-mapping $T$.

As a correction of Theorem 1, we should state

Theorem 2. Let $(X, \rho)$ be a complete metric space. A self-mapping $T: X \rightarrow X$ possesses a fixed point in $X$, if there exist constants $\lambda \in[0,1)$ and $\alpha \in(0,1)$ such that

$$
\rho(T \xi, T \eta) \leq \lambda[\rho(\xi, T \xi)]^{\alpha} \cdot[\rho(\eta, T \eta)]^{1-\alpha}
$$

for all $\xi, \eta \in X \backslash \operatorname{Fix}(T)$.

The following theorem was proved by Reich, Rus and Ćirić [3-7] independently to combine and improve both Banach and Kannan fixed point theorems.

Theorem 3. In the framework of a complete metric space $(X, \rho)$, if $T: X \rightarrow X$ forms a Reich-Rus-Ćirić contraction mapping, i.e.,

$$
\rho(T \xi, T \eta) \leq \lambda[\rho(\xi, \eta)+\rho(\xi, T \xi)+\rho(\eta, T \eta)]
$$

for all $\xi, \eta \in X$, where $\lambda \in\left[0, \frac{1}{3}\right)$, then $T$ possesses a unique fixed point.

Notice that several variations of Reich contractions (3) can be stated. We may state the following:

$$
\rho(T \xi, T \eta) \leq a \rho(\xi, \eta)+b \rho(\xi, T \xi)+c \rho(\eta, T \eta),
$$

where $a, b, c \in(0, \infty)$ such that $0 \leq a+b+c<1$.

In this paper, we shall investigate the validity of the interpolation approach for Reich contractions in the context of partial metric spaces that was introduced by Matthews [8].

Definition 1. Let $X$ be a non-empty set. A function $p: X \times X \rightarrow[0, \infty)$ is said to be a partial metric, if the following conditions are fulfilled for each $\xi, \eta, \zeta \in X$,

$$
\begin{aligned}
& (P 1) \quad \xi=\eta \Leftrightarrow p(\xi, \xi)=p(\eta, \eta)=p(\xi, \eta) \\
& (P 2) \quad p(\xi, \xi) \leq p(\xi, \eta) \\
& (P 3) \quad p(\xi, \eta)=p(\eta, \xi) \\
& (P 4) \quad p(\xi, \eta) \leq p(\xi, \zeta)+p(\zeta, \eta)-p(\zeta, \zeta) .
\end{aligned}
$$

In this case, $(X, p)$ is said to be a partial metric space. 
The function $\rho_{p}: X \times X \rightarrow[0, \infty)$ defined as

$$
\rho_{p}(\xi, \eta)=2 p(\xi, \eta)-p(\xi, \xi)-p(\eta, \eta)
$$

is a standard metric on $X$. It is natural to define the basic topological concepts, in particular, convergence of a sequence, fundamental (Cauchy) sequence criteria, continuity of the mappings, and completeness of the topological space in the framework of partial metric spaces; see, e.g., [8-18].

Definition 2. In the framework of a partial metric space $(X, p)$, we say that

(i) A sequence $\left\{\xi_{n}\right\}$ converges to the limit $\xi$ if $p(\xi, \xi)=\lim _{n \rightarrow \infty} p\left(\xi, \xi_{n}\right)$;

(ii) A sequence $\left\{\xi_{n}\right\}$ is fundamental or Cauchy if $\lim _{n, m \rightarrow \infty} p\left(\xi_{n}, \xi_{m}\right)$ exists and is finite;

(iii) A partial metric space $(X, p)$ is complete if each fundamental sequence $\left\{\xi_{n}\right\}$ converges to a point $\xi \in X$ such that $p(\xi, \xi)=\lim _{n, m \rightarrow \infty} p\left(\xi_{n}, \xi_{m}\right)$;

(iv) A mapping $F: X \rightarrow X$ is continuous at a point $\xi_{0} \in X$ if for each $\epsilon>0$, there exists $\delta>0$ such that $F\left(B_{p}\left(\xi_{0}, \delta\right)\right) \subseteq B_{P}\left(F \xi_{0}, \epsilon\right)$.

For what follows, we shall recall the following lemma that can be derived easily (see [8]).

Lemma 1. Let $p$ be a partial metric on a non-empty set $X$ and $\rho_{p}$ be the corresponding standard metric space on the same set $X$.

(a) A sequence $\left\{\xi_{n}\right\}$ is fundamental in the framework of a partial metric $(X, p)$ if and only if it is a fundamental sequence in the setting of the corresponding standard metric space $\left(X, \rho_{p}\right)$.

(b) A partial metric space $(X, p)$ is complete if and only if the corresponding standard metric space $\left(X, \rho_{p}\right)$ is complete. Moreover,

$$
\lim _{n \rightarrow \infty} \rho_{p}\left(\xi_{,} \xi_{n}\right)=0 \Leftrightarrow p(\xi, \xi)=\lim _{n \rightarrow \infty} p\left(\xi_{,} \xi_{n}\right)=\lim _{n, m \rightarrow \infty} p\left(\xi_{n}, \xi_{m}\right) .
$$

(c) If $\xi_{n} \rightarrow \zeta$ as $n \rightarrow \infty$ in a partial metric space $(X, p)$ with $p(\zeta, \zeta)=0$, then we have

$$
\lim _{n \rightarrow \infty} p\left(\xi_{n}, \eta\right)=p(\zeta, \eta) \text { for every } \eta \in X
$$

In this paper, we initiate the notion of interpolative Reich-Rus-Ćirić type contractions on partial metric spaces. We also present two examples illustrating our approach.

\section{Main Results}

We start this section by introducing the notion of interpolative Reich-Rus-Ćirić type contractions.

Definition 3. In the framework of a partial metric space $(X, p)$, a mapping $T: X \rightarrow X$ is called an interpolative Reich-Rus-Ćirić type contraction, if there are constants $\lambda \in[0,1)$ and $\alpha, \beta \in(0,1)$ such that

$$
p(T \xi, T \eta) \leq \lambda[p(\xi, \eta)]^{\beta} \cdot[p(\xi, T \xi)]^{\alpha} \cdot[p(\eta, T \eta)]^{1-\alpha-\beta}
$$

for all $\xi, \eta \in X \backslash F i x(T)$,

Theorem 4. In the framework of a partial metric space $(X, p)$, if $T: X \rightarrow X$ is an interpolative Reich-Rus-Ćirić type contraction, then $T$ has a fixed point in $X$.

Proof. We take an arbitrary point $\xi_{0} \in(X, p)$ and build an iterative sequence $\left\{\xi_{n}\right\}$ by $\xi_{n}=T^{n}\left(\xi_{0}\right)$ for each positive integer $n$. If there exists $n_{0}$ such that $\xi_{n_{0}}=\xi_{n_{0}+1}$, then $\xi_{n_{0}}$ is a fixed point of $T$. The proof 
is completed. Henceforwards, assume that $\xi_{n} \neq \xi_{n+1}$ for each $n \geq 0$. By substituting the values $\xi=\xi_{n}$ and $\eta=\xi_{n-1}$ in (7), we find that

$$
\begin{aligned}
p\left(\xi_{n+1}, \xi_{n}\right)=p\left(T \xi_{n}, T \xi_{n-1}\right) & \leq \lambda\left[p\left(\xi_{n}, \xi_{n-1}\right)\right]^{\beta}\left[p\left(\xi_{n}, T \xi_{n}\right)\right]^{\alpha} \cdot\left[p\left(\xi_{n-1}, T \xi_{n-1}\right)\right]^{1-\alpha-\beta} \\
& =\lambda\left[p\left(\xi_{n}, \xi_{n-1}\right)\right]^{\beta} \cdot\left[p\left(\xi_{n}, \xi_{n+1}\right)\right]^{\alpha} \cdot\left[p\left(\xi_{n-1}, \xi_{n}\right)\right]^{1-\alpha-\beta} \\
& =\lambda\left[p\left(\xi_{n-1}, \xi_{n}\right)\right]^{1-\alpha} \cdot\left[p\left(\xi_{n}, \xi_{n+1}\right)\right]^{\alpha} .
\end{aligned}
$$

By a calculation, we derive

$$
\left[p\left(\xi_{n}, \xi_{n+1}\right)\right]^{1-\alpha} \leq \lambda\left[p\left(\xi_{n-1}, \xi_{n}\right)\right]^{1-\alpha}
$$

from the inequality (8). We conclude that $\left\{p\left(\xi_{n-1}, \xi_{n}\right)\right\}$ is a non-increasing sequence with non-negative terms. Thus, there is a nonnegative constant $\ell$ such that $\lim _{n \rightarrow \infty} p\left(\xi_{n-1}, \xi_{n}\right)=\ell$. Note that $\ell \geq 0$. Indeed, from (9), we deduce that

$$
p\left(\xi_{n}, \xi_{n+1}\right) \leq \lambda p\left(\xi_{n-1}, \xi_{n}\right) \leq \lambda^{n} p\left(\xi_{0}, \xi_{1}\right)
$$

Regarding $\lambda<1$, and by taking $n \rightarrow \infty$ in the inequality (10), we deduce that $\ell=0$.

For what follows, we shall prove that $\left\{\xi_{n}\right\}$ is a fundamental (Cauchy) sequence by employing standard tools. More precisely, starting with the triangle inequality, we shall get the following estimation:

$$
\begin{aligned}
p\left(\xi_{n}, \xi_{n+r}\right) & \leq p\left(\xi_{n}, \xi_{n+1}\right)+\cdots+p\left(\xi_{n+r-1}, \xi_{n+r}\right) \\
& \leq \lambda^{n} p\left(\xi_{0}, \xi_{1}\right)+\cdots+\lambda^{n+r-1} p\left(\xi_{0}, \xi_{1}\right) \\
& \leq \frac{\lambda^{n}}{1-\lambda} p\left(\xi_{0}, \xi_{1}\right) .
\end{aligned}
$$

Letting $n \rightarrow \infty$ in the inequality (11), we ascertain that $\left\{\xi_{n}\right\}$ is a fundamental sequence.

Hence, $\lim _{n, m \rightarrow \infty} p\left(\xi_{n}, \xi_{m}\right)=0$, that is, $\left\{\xi_{n}\right\}$ is a fundamental sequence in $(X, p)$. By Lemma 1 , $\left\{\xi_{n}\right\}$ is also Cauchy in $\left(X, \rho_{p}\right)$. More particularly, since $(X, p)$ is complete, $\left(X, \rho_{p}\right)$ is also complete. Hence, there exists $\xi \in X$ such that

$$
p(\xi, \xi)=\lim _{n \rightarrow \infty} p\left(\xi, \xi_{n}\right)=\lim _{n, m \rightarrow \infty} p\left(\xi_{n}, \xi_{m}\right)=0,
$$

which implies that

$$
\lim _{n \rightarrow \infty} \rho_{p}\left(\xi, \xi_{n}\right)=0 .
$$

As a next step, we make evident that the limit $\xi$ of the iterative sequence $\left\{\xi_{n}\right\}$ is a fixed point of the given mapping $T$. Assume that $\xi \neq T \xi$, so $p(\xi, T \xi)>0$. Recall that $x_{n} \neq T x_{n}$ for each $n \geq 0$. By letting $\xi=\xi_{n}$ and $\eta=\xi$ in (7), we determine that

$$
\begin{aligned}
p\left(\xi_{n+1}, T \xi\right) & =p\left(T \xi_{n}, T \xi\right) \\
& \leq \lambda\left[p\left(\xi_{n}, \xi\right)\right]^{\beta} \cdot\left[p\left(\xi_{n}, T \xi_{n}\right)\right]^{\alpha} \cdot[p(\xi, T \xi)]^{1-\alpha-\beta} \\
& =\lambda\left[p\left(\xi_{n}, \xi\right)\right]^{\beta} \cdot\left[p\left(\xi_{n}, \xi_{n+1}\right)\right]^{\alpha} \cdot[p(\xi, T \xi)]^{1-\alpha-\beta} .
\end{aligned}
$$

Letting $n \rightarrow \infty$ in the inequality (14), we find out $p(\xi, T \xi)=0$, so $\xi=T \xi$, which is a contradiction. Thus, $T \xi=\xi$. 
The following examples illustrate Theorem 4.

Example 2. Let $X=\{1,3,4,7\}$ be a set endowed with the classical partial metric $\rho(\xi, \eta)=\max \{\xi, \eta\}$, that is,

\begin{tabular}{|c|c|c|c|c|}
\hline$\rho(\xi, \eta)$ & 1 & 3 & 4 & 7 \\
\hline 1 & 1 & 3 & 4 & 7 \\
\hline 3 & 3 & 3 & 4 & 7 \\
\hline 4 & 4 & 4 & 4 & 7 \\
\hline 7 & 7 & 7 & 7 & 7 \\
\hline
\end{tabular}

We define a self-mapping $T$ on $X$ by $T:\left(\begin{array}{llll}1 & 3 & 4 & 7 \\ 1 & 3 & 1 & 3\end{array}\right)$. It is clear that $T$ is not a Reich-Rus-Ćirić contraction. Indeed, there is no $\lambda \in\left[0, \frac{1}{3}\right)$ such that the following inequality is fulfilled:

$$
\begin{aligned}
\rho(T 1, T 3)=\rho(1,3)=3 & \leq \lambda(\rho(1,3)+\rho(T 1,1)+\rho(3, T 3)) \\
& =\lambda(\rho(1,3)+\rho(1,1)+\rho(3,3)) \\
& =7 \lambda
\end{aligned}
$$

On the other hand, choose $\alpha=\frac{1}{2}, \beta=\frac{2}{5}$ and $\lambda=\frac{7}{10}$. Let $\xi, \eta \in X \backslash$ Fix $(T)$; then, $(\xi, \eta) \in$ $\{(4,7),(7,4),(4,4),(7,7)\}$. Without loss of generality, we have

Case 1: $\xi=\eta=4$. Here,

$$
\rho(T \xi, T \eta)=1 \leq 4 \lambda=\lambda[\rho(\xi, y)]^{\beta} \cdot[\rho(\xi, T \xi)]^{\alpha} \cdot[\rho(\eta, T \eta)]^{1-\alpha-\beta} .
$$

Case 2: $\xi=\eta=7$. we have

$$
\rho(T \xi, T \eta)=3 \leq 7 \lambda=\lambda[\rho(\xi, y)]^{\beta} \cdot[\rho(\xi, T \xi)]^{\alpha} \cdot[\rho(\eta, T \eta)]^{1-\alpha-\beta} .
$$

Case 3: $\xi=4$ and $\eta=7$. Here,

$$
\begin{aligned}
\rho(T \xi, T \eta) & =3 \leq \lambda 7^{1-\alpha} 4^{\alpha} \\
& =\lambda[\rho(\xi, y)]^{\beta} \cdot[\rho(\xi, T \xi)]^{\alpha} \cdot[\rho(\eta, T \eta)]^{1-\alpha-\beta} .
\end{aligned}
$$

Thus, the self-mapping $T$ is an interpolative Reich-Rus-Ćirić type contraction and 1, 3 are the desired fixed points. Note that, in the setting of interpolative Reich-Rus-Ćirić type contractions, the constant lies between 0 and 1 , although in the classical version it is restricted by $1 / 3$.

Example 3. Following Example 1, let $X=\{0,1,2,3\}$. Consider

$$
T 0=0, \quad T 1=1, \quad T 2=T 3=1 .
$$

Consider $p(x, y)=\max \{x, y\}$. Clearly, (7) holds for all $\xi, \eta \in X \backslash$ Fix $(T)$ [by taking $\lambda=\frac{\sqrt{2}}{2}, \beta=\frac{1}{2}$ and $\alpha=\frac{1}{3}$ ]. Note that $T$ has two fixed points, which are 0 and 1 .

On the other hand, taking $x=0$ and $y=1$, we have, for any $\lambda \in\left[0, \frac{1}{3}\right)$,

$$
p(T x, T y)=1>2 \lambda=\lambda[p(x, y)+p(x, T x)+p(y, T y)]
$$

that is, Corollary 4 in [9] is not applicable.

The following is an immediate consequence of our main result. 
Corollary 1. In the framework of a standard metric space $(X, \rho)$, if $T: X \rightarrow X$ is an interpolative Reich-Rus-Cirić type contraction, that is,

$$
\rho(T \xi, T \eta) \leq \lambda[\rho(\xi, y)]^{\beta} \cdot[\rho(\xi, T \xi)]^{\alpha} \cdot[\rho(\eta, T \eta)]^{1-\alpha-\beta}
$$

for all $\xi, \eta \in X \backslash$ Fix $(T)$, then $T$ possesses a fixed point in $X$.

Proceeding as Theorem 4, we shall extend Theorem 2 to partial metric spaces.

Theorem 5. Let $(X, p)$ be a complete partial metric space and $T: X \rightarrow X$ be such that

$$
p(T \xi, T \eta) \leq \lambda \cdot[p(\xi, T \xi)]^{\alpha} \cdot[p(x, T \eta)]^{1-\alpha}
$$

for all $\xi, \eta \in X \backslash F i x(T)$, where $\lambda \in[0,1)$ and $\alpha \in(0,1)$. Then, $T$ possesses a fixed point in $X$.

\section{Conclusions}

The main contribution of the paper to ensure the existence of fixed points for interpolative Reich-Rus-Ćirić type contraction mappings on partial metric spaces. We also removed the gap in [2].

Author Contributions: All authors contributed equally and significantly in writing this article. All authors read and approved the final manuscript.

Funding: This research received no external funding.

Conflicts of Interest: The authors declare that they have no competing interests.

\section{References}

1. Kannan, R. Some results on fixed points. Bull. Calcutta Math. Soc. 1968, 60, 71-76.

2. Karapinar, E. Revisiting the Kannan Type Contractions via Interpolation. Adv. Theory Nonlinear Anal. Appl. 2018, 2, 85-87. [CrossRef]

3. Reich, S. Some remarks concerning contraction mappings. Can. Math. Bull. 1971, 14, 121-124. [CrossRef]

4. Reich, S. Fixed point of contractive functions. Boll. Unione Mat. Ital. 1972, 4, 26-42.

5. Reich, S. Kannan's fixed point theorem. Boll. Unione Mat. Ital. 1971, 4, 1-11.

6. Rus, I.A. Principles and Applications of the Fixed Point Theory; Editura Dacia: Clui-Napoca, Romania, 1979. (In Romanian)

7. Rus, I.A. Generalized Contractions and Applications; Cluj University Press: Clui-Napoca, Romania, 2001.

8. Matthews, S.G. Partial metric topology. Ann. N. Y. Acad. Sci. 1994, 728, 183-197. [CrossRef]

9. Altun, I.; Sola, F.; Simsek, H. Generalized contractions on partial metric spaces. Topol. Appl. 2010, 157, 2778-2785. [CrossRef]

10. Aydi, H.; Karapinar, E.; Kumam, P. A Note on "[Modied Proof of Caristi's Fixed Point Theorem on Partial Metric Spaces, Journal of Inequalities and Applications 2013, 2013:210]". J. Inequal. Appl. 2013. [CrossRef]

11. Aydi, H.; Amor, S.H.; Karapinar, E. Berinde Type generalized contractions on partial metric spaces. Abstr. Appl. Anal. 2013, 2013, 312479. [CrossRef]

12. Aydi, H.; Karapinar, E.; Shatanawi, W. Coupled fixed point results for $(\phi)$-weakly contractive condition in ordered partial metric spaces. Comput. Math. Appl. 2011, 62, 4449-4460. [CrossRef]

13. Aydi, H.; Karapinar, E.; Rezapour, S. A generalized Meir-Keeler contraction type on partial metric spaces. Abstr. Appl. Anal. 2012, 2012, 287127. [CrossRef]

14. Aydi, H.; Karapinar, E. A Meir-Keeler common type fixed point theorem on partial metric spaces. Fixed Point Theory Appl. 2012, 2012, 26. [CrossRef]

15. Ćirić, L.J.; Samet, B.; Aydi, H.; Vetro, C. Common fixed points of generalized contractions on partial metric spaces and an application. Appl. Math. Comput. 2011, 218, 2398-2406. [CrossRef]

16. Chi, K.P.; Karapinar, E.; Thanh, T.D. A Generalized contraction principle in partial metric spaces. Math. Comput. Model. 2012, 55, 1673-1681. [CrossRef] 
17. Karapinar, E.; Erhan, Y.M.; Ulus, A.Y. Fixed Point Theorem for Cyclic Maps on Partial Metric Spaces. Appl. Math. Inf. Sci. 2012, 6, 239-244.

18. Karapinar, E.; Chi, K.P.; Thanh, T.D. A generalization of Ciric quasi-contractions. Abstr. Appl. Anal. 2012, 2012. [CrossRef]

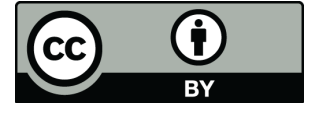

(C) 2018 by the authors. Licensee MDPI, Basel, Switzerland. This article is an open access article distributed under the terms and conditions of the Creative Commons Attribution (CC BY) license (http:/ / creativecommons.org/licenses/by/4.0/). 